\title{
A Novel Integral Operator Transform and Its Application to Some FODE and FPDE with Some Kind of Singularities
}

\author{
Abdon Atangana ${ }^{1}$ and Adem Kilicman ${ }^{2}$ \\ ${ }^{1}$ Institute for Groundwater Studies, Faculty of Natural and Agricultural Sciences, University of the Free State, \\ Bloemfontein 9300, South Africa \\ ${ }^{2}$ Department of Mathematics and Institute for Mathematical Research, Universiti Putra Malaysia, 43400, Serdang, Selangor, Malaysia
}

Correspondence should be addressed to Adem Kilicman; kilicman@yahoo.com

Received 14 April 2013; Accepted 11 July 2013

Academic Editor: Hossein Jafari

Copyright (C) 2013 A. Atangana and A. Kilicman. This is an open access article distributed under the Creative Commons Attribution License, which permits unrestricted use, distribution, and reproduction in any medium, provided the original work is properly cited.

We introduced a novel integral transform operator. We proved the existence and the uniqueness of the relatively new operator. We presented some useful properties of the new operator. We presented the application of this operator for solving some kind of fractional ordinary and partial differential equation containing some kind of singularity.

\section{Introduction}

Mathematical notation aside, the motivation behind integral transforms is easy to understand. There are many classes of problems that are difficult to solve or at least quite unwieldy algebraically in their original representations. An integral transform "maps" an equation from its original "domain" into another domain [1-3]. Manipulating and solving the equation in the target domain can be much easier than manipulation and solution in the original domain. The solution is then mapped back to the original domain with the inverse of the integral transform. There exist few integral transform operators in the literature [1-3], which are commonly used to solve partial fractional and fractional ordinary differential equations.

The Fourier transform, named after Joseph Fourier, is a mathematical transform with many applications in physics and engineering [4-11]. Very commonly it transforms a mathematical function of time, $f(t)$, into a new function, sometimes denoted by $F$, whose argument is frequency with units of cycles per second or (hertz) or radians per second. The new function is then known as the Fourier transform and/or the frequency spectrum of the function $f$. The Fourier transform is also a reversible operation. Thus, given the function $\hat{f}$ one can determine the original function $f$; see in [8].
The Laplace transform is an integral transform perhaps second only to the Fourier transform in its utility in solving physical problems [12-17]. The Laplace transform is particularly useful in solving linear ordinary differential equations or partial fractional differential equations such as those arising in the analysis of groundwater pollution model [13] and electronic circuits [14].

In mathematics, the Mellin transform [15] is an integral transform that may be regarded as the multiplicative version of the two-sided Laplace transform. This integral transform is closely connected to the theory of Dirichlet series and is often used in number theory and the theory of asymptotic expansions; it is closely related to the Laplace transform and the Fourier transform and the theory of the gamma function and allied special functions.

The Mellin transform is widely used in computer science because of its scale invariance property [18]. The magnitude of the Mellin transform of a scaled function is identical to the magnitude of the original function [18]. This scale invariance property is analogous to the Fourier transform's shift invariance property. The magnitude of a Fourier transform of a time-shifted function is identical to the original function. This property is useful in image recognition. An image of an object is easily scaled when the object is moved towards or away from the camera [19]. 
In mathematics, the Sumudu transform is an integral transform similar to the Laplace transform, introduced in the early 1990s by Watugala to solve differential equations and control engineering problems [20-27]. It is equivalent to the Laplace-Carson transform with the substitution $p=1 / u$.

However, there exists some kind of fractional ordinary and partial differential equations with some kind of singularities that cannot be solved directly via the above integral transform operators. In particular, the following kind of fractional ordinary and partial differential equations

$$
{ }_{0} D_{x}^{\alpha} y(x)+\frac{1}{x^{n}} y(x)=f(x)
$$

or

$$
{ }_{0} D_{x}^{\alpha} u(x, t)+\frac{1}{x^{n}} u(x, t)=h(x, t)
$$

where $\alpha>0,{ }_{0} D_{x}^{\alpha}$, is the fractional derivative (RiemannLiouville or Caputo) and $n \geq 1$.

To solve the above equations, some scholars make use of the Frobenius method, to obtain the solutions in series form. The Laplace transform of the product of two functions is different from the product of the Laplace transform of the two functions. The Fourier transform of the product of two functions is equivalent to the convolution of the Fourier transform of the two functions. This renders it very difficult to apply directly either the Laplace transform or the Fourier transform operators to solve this type of equation. Therefore some scholars multiply $x^{n}$ on both sides of the above equations and then apply the Fourier or the Laplace transform. It is therefore worth to define an integral transform similar to Laplace or Laplace-Carson transform to transform such equation to an ordinary or partial differential equation without any additional transformation.

The aim of this work is to further introduce an integral transform operator that can be used to solve some kind of ordinary, partial and fractional ordinary, partial differential equation with some kind of singularities. We will start with the definition and present some theorems.

\section{Definitions and Theorems}

Definition 1. Let $f(x)$ be a continuous function over an open interval $(0, \infty)$ such that its Laplace transform is $n$ time differentiable; then, the new integral transform of order $n$ of $f$ is defined as follows:

$$
M_{n}(s)=M_{n}[f(x)](s)=\int_{0}^{\infty} x^{n} e^{-x s} f(x) d x
$$

and the inverse of the new integral transform of order $n$ is defined as

$$
\begin{aligned}
f(x)= & M_{n}^{-1}\left[M_{n}[f(x)]\right] \\
=\frac{(-1)^{n}}{2 \pi i} \int_{\alpha-i \infty}^{\alpha+i \infty} e^{s x}\left[(-1)^{n}[\right. & \frac{1}{\Gamma(n-1)} \\
& \times \int_{0}^{s}(s-t)^{n-1} M_{n}(t) d t \\
& \left.\left.+\sum_{k=0}^{n-1} \frac{s^{k}}{k !} y_{k}\right]\right] d s, \\
& y_{k}=\frac{\partial^{k} F(0)}{\partial s^{k}},
\end{aligned}
$$

where $F(s)$ is the Laplace transform of $f(x)$. Before we continue, we will prove that the above definition is indeed the inverse operator transform of order $n$. In fact from the definition of new transform of order $n$ of a function $f(x)$, we have that

$$
\begin{aligned}
M_{n}(s) & =M_{n}[f(x)](s) \\
& =\int_{0}^{\infty} x^{n} e^{-x s} f(x) d x=(-1)^{n} \frac{d^{n} F(s)}{d s^{n}},
\end{aligned}
$$

thus

$$
\begin{gathered}
\frac{1}{\Gamma(n-1)} \int_{0}^{s}(s-t)^{n-1} M_{n}(t) d t \\
=(-1)^{n}\left[F(s)-\sum_{k=0}^{n-1} \frac{s^{k}}{k !} y_{k}\right] .
\end{gathered}
$$

It follows that

$$
\begin{gathered}
\frac{(-1)^{n}}{2 \pi i} \int_{\alpha-i \infty}^{\alpha+i \infty} e^{s x}\left[( - 1 ) ^ { n } \left[\frac{1}{\Gamma(n-1)}\right.\right. \\
\quad \times \int_{0}^{s}(s-t)^{n-1} M_{n}(t) d t \\
\left.\left.\quad+\sum_{k=0}^{n-1} \frac{s^{k}}{k !} y_{k}\right]\right] d s \\
=\frac{(-1)^{n}}{2 \pi i} \int_{\alpha-i \infty}^{\alpha+i \infty} e^{s x}\left[(-1)^{n}[F(s)]\right] d s \\
M_{n}^{-1}\left[M_{n}[f(x)]\right] \\
=\frac{(-1)^{2 n}}{2 \pi i} \int_{\alpha-i \infty}^{\alpha+i \infty} e^{s x}[[F(s)]] d s=f(x) .
\end{gathered}
$$

Therefore the inverse of the new integral transform is well defined. Our next concern is to prove the uniqueness and the existence of the new integral transform. 
Theorem 2. Let $f(x)$ and $g(x)$ be continuous functions defined for $x \geq 0$ and having new transforms of order $n, F(p)$, and $G(p)$, respectively. If $F(p)=G(p)$, then $f(x)=g(x)$.

Proof. From the definition of the inverse of the new transform of order $n$, if $\alpha$ is sufficiently large, then the integral expression, by

$$
f(x)=\frac{(-1)^{n}}{2 \pi i} \int_{\alpha-i \infty}^{\alpha+i \infty} e^{p x}\left[(-1)^{n} F(p)\right] d p
$$

for the inverse of the new integral transform of order $n$, can be used to obtain

$$
f(x)=\frac{(-1)^{2 n}}{2 \pi i} \int_{\alpha-i \infty}^{\alpha+i \infty} e^{p x}[F(p)] d p .
$$

By hypothesis we have that $F(p)=G(p)$; then replacing this in the above expression we have the following:

$$
f(x)=\frac{(-1)^{n}}{2 \pi i} \int_{\alpha-i \infty}^{\alpha+i \infty} e^{p x}\left[(-1)^{n} G(p)\right] d p
$$

which boils down to

$$
f(x)=\frac{(-1)^{n}}{2 \pi i} \int_{\alpha-i \infty}^{\alpha+i \infty} e^{p x}\left[(-1)^{n} G(p)\right] d p=g(x)
$$

and this proves the uniqueness of the new integral transform of order $n$.

Theorem 3. If $f(t)$ is a piecewise continuous on every finite interval in $\left[0, t_{0}\right)$ and satisfies:

$$
\left|t^{n} f(t)\right| \leq M e^{\alpha t}
$$

for all $t \in\left[t_{0}, \infty\right)$, then $M_{n}[f(x)](s)$ exists for all $s>\alpha$.

Proof. To prove the theorem we must show that the improper integral converges for $s>a$. Splitting the improper integral into two parts, we have

$$
\begin{aligned}
\int_{0}^{\infty} t^{n} & e^{-s t} f(t) d t \\
& =\int_{0}^{t_{0}} t^{n} e^{-s t} f(t) d t+\int_{t_{0}}^{\infty} t^{n} e^{-s t} f(t) d t
\end{aligned}
$$

The first integral on the right side exists by hypothesis 1; hence the existence of the new integral transform of order $n$, $M_{n}(s)$, depends on the convergence of the second integral. By hypothesis 2 , we have

$$
\left|t^{n} e^{-s t} f(t)\right| \leq M e^{\alpha t} e^{-s t}=M e^{(\alpha-s) t}
$$

Now

$$
\int_{t_{0}}^{\infty} M e^{(\alpha-s) t} d t=M \frac{e^{(\alpha-s) t_{0}}}{\alpha-s}
$$

this converges for $\alpha<s$. Then, by the comparison test for improper integrals theorem, $M_{n}(s)$, exists for $\alpha<s$.
Remark 4. There is a relationship between the Laplace transform and the new integral transform of order $n$ as follows:

$$
\begin{gathered}
L(f(x))(s)=M_{n}\left(\frac{1}{x^{n}} f(x)\right)(s), \\
L(f(x))(s)=M_{0}(f(x))(s), \\
M_{n}(f(x))(s)=(-1)^{n} \frac{d^{n}[F(s)]}{d s^{n}},
\end{gathered}
$$

where $F(s)$ is the Laplace transform of $f(x)$.

Remark 5. There is a relationship between the LaplaceCarson transform and then new integral transform of order $n$ as follows:

$$
L_{c}(f(x))(s)=M_{1}(f(x))(s) .
$$

Theorem 6. A function $f(x)$ which is continuous on $[0, \infty)$ and satisfies the growth condition $f(x)$ can be recovered from the Laplace transform $F(p)$ as follows:

$$
f(x)=\lim _{n \rightarrow \infty} \frac{(-1)^{n}}{n !}\left(\frac{n}{x}\right)^{n+1} M_{n}\left(\frac{n}{s}\right) .
$$

Evidently, the main difficulty in using Theorem 6 for computing the inverse Laplace transform is the repeated symbolic differentiation of $F(p)$.

\section{Some Properties of the New Integral Transform}

In this section, we consider some of the properties of the new integral transform that will enable us to find further transform pairs $\left\{f(x), M_{n}(s)\right\}$ without having to compute consider the following:

(I) $M_{n}[s+c]=M_{n}\left[e^{-c x} f(x)\right]$,

(II) $M_{n}[f(a x)](s)=\frac{1}{a} M_{n}\left[\frac{s}{a}\right]$,

(III) $\int_{\alpha-i \infty}^{\alpha+i \infty} e^{s x} M_{n}(s) d s=x^{n} f(x)$,

(IV) $M_{n}[a f(x)+b g(x)](s)$

$$
=\left[a M_{n}(f(x))+b M_{n}(g(x))\right](s)
$$

(V) $M_{n}\left[\frac{f(x)}{x^{n}}\right](s)=L[f(x)](s)$,

(VI) $M_{n}[f(x) * h(x)](s)$

$$
=(-1)^{n} \sum_{k=0}^{n} C_{n}^{k} \frac{d^{k}(G(s))}{d s^{k}} \times \frac{d^{n-k}(F(s))}{d s^{n-k}}
$$

$$
\text { (VII) } \begin{aligned}
& M_{n}\left[\frac{d^{n} f(x)}{d x^{n}}\right](s) \\
= & (-1)^{n} \sum_{k=0}^{n} C_{n}^{k} \frac{d^{k}\left(s^{n}\right)}{d s^{k}} \times \frac{d^{n-k}(F(s))}{d s^{n-k}} .
\end{aligned}
$$


Let us verify the above properties. We will start with I, by definition we have the following:

$$
\begin{aligned}
M_{n}\left[e^{-c x} f(x)\right] \\
\quad=\int_{0}^{\infty}\left[x^{n} e^{-c x} e^{-s x} f(x)\right] d x \\
=\int_{0}^{\infty}\left[x^{n} e^{-(c+s) x} f(x)\right] d x=M_{n}[s+c]
\end{aligned}
$$

and then the first property is verified.

For II we have the following by definition:

$$
\begin{aligned}
M_{n}[ & f(a x)](s) \\
& =\int_{0}^{\infty}\left[x^{n} e^{-x s} f(a x)\right] d x=(-1)^{n} \frac{d^{n}}{d s^{n}}[L[f(a x)](s)] .
\end{aligned}
$$

Now using the property of the Laplace transform $L[f(a x)](s)=(1 / a) F(s / a)$, from this we can further obtain

$$
\begin{aligned}
M_{n}[ & f(a x)](s) \\
& =(-1)^{n} \frac{d^{n}}{d s^{n}}\left[\frac{1}{a} F\left(\frac{s}{a}\right)\right] \\
& =\frac{1}{a}(-1)^{n} \frac{d^{n}}{d s^{n}}\left[F\left(\frac{s}{a}\right)\right]=\frac{1}{a} M_{n}\left[\frac{s}{a}\right]
\end{aligned}
$$

and then, the property number II is verified.

For number III we have the following: Let $g(x)=x^{n} f(x)$, then

$$
\begin{aligned}
\int_{\alpha-i \infty}^{\alpha+i \infty} & e^{s x} M_{n}(s) d s \\
& =\int_{\alpha-i \infty}^{\alpha+i \infty} e^{s x}\left[\int_{0}^{\infty} e^{-x s} x^{n} f(x) d x\right] d s \\
& =\int_{\alpha-i \infty}^{\alpha+i \infty} e^{s x}\left[\int_{0}^{\infty} e^{-x s} g(x) d x\right] d s .
\end{aligned}
$$

By the theorem of inverse Laplace transform we obtain

$$
\int_{\alpha-i \infty}^{\alpha+i \infty} e^{s x} M_{n}(s) d s=g(x)=x^{n} f(x)
$$

numbers IV and V are obvious to be verified. For number VI we have the following by definition:

$$
\begin{aligned}
M_{n}[f(x) * h(x)](s) \\
\quad=\int_{0}^{\infty}\left[x^{n} e^{-s x} f(x) * h(x)\right] \\
=(-1)^{n} \frac{d^{n}}{d s^{n}}[L(f(x) * h(x))(s)],
\end{aligned}
$$

now using the property of Laplace transform of the convolution, we obtain the following:

$$
L(f(x) * h(x))(s)=F(s) \cdot G(s)
$$

and then, using the property of the derivative of order $n$ for the product of two functions, we obtain

$$
\begin{aligned}
M_{n}[ & f(x) * h(x)](s) \\
& =(-1)^{n} \frac{d^{n}}{d s^{n}}[F(s) \cdot G(s)] \\
& =(-1)^{n} \sum_{k=0}^{n} C_{n}^{k} \frac{d^{k}(G(s))}{d s^{k}} \times \frac{d^{n-k}(F(s))}{d s^{n-k}}
\end{aligned}
$$

and then, the property number VI is verified.

For number VII, by definition, we have the following:

$$
\begin{aligned}
M_{n}[ & \left.\frac{d^{n} f(x)}{d x^{n}}\right](s) \\
& =\int_{0}^{\infty}\left[x^{n} e^{-s x} \frac{d^{n} f(x)}{d x^{n}}\right] d x \\
& =(-1)^{n} \frac{d^{n}}{d s^{n}}\left[L\left(\frac{d^{n} f(x)}{d x^{n}}\right)(s)\right],
\end{aligned}
$$

now using the property of the Laplace transform,

$$
L\left(\frac{d^{n} f(x)}{d x^{n}}\right)(s)=s^{n} F(s)-\sum_{k=0}^{n-1} s^{n-k-1} \frac{d^{k} f(0)}{d x^{k}}
$$

now deriving the above expression $n$ times, we obtain the following expression:

$$
\begin{aligned}
& (-1)^{n} \frac{d^{n}}{d s^{n}}\left[L\left(\frac{d^{n} f(x)}{d x^{n}}\right)(s)\right] \\
& =(-1)^{n} \sum_{k=0}^{n} C_{n}^{k} \frac{d^{k}\left(s^{n}\right)}{d s^{k}} \times \frac{d^{n-k}(F(s))}{d s^{n-k}}
\end{aligned}
$$

that is:

$$
M_{n}\left[\frac{d^{n} f(x)}{d x^{n}}\right](s)=(-1)^{n} \sum_{k=0}^{n} C_{n}^{k} \frac{d^{k}\left(s^{n}\right)}{d s^{k}} \times \frac{d^{n-k}(F(s))}{d s^{n-k}} .
$$

This completes the proof of number VI.

\section{Application to FODE and FPDE}

Recently, the differential equations of fractional order derivative with singularities have been the focus of many studies due to their frequent appearance in various applications in fluid mechanics, viscoelasticity, biology physics, engineering, and groundwater models, in particular the monitoring of the flow through the geological formation and the pollution migration. Consequently, considerable attention has been given to the solutions of fractional differential equations and integral equations with singularity of physical interest. There exists in the literature some integral transform method that can be used to derive exact and approximate solutions for such equations; see, for instance, Laplace transform method [4-11], the Fourier transform method [12-17], the Mellin 
transform method $[18,19]$, the Sumudu transform method [20-27], the Adomian decomposition method [28, 29], and the homotopy decomposition method [30-33]. In this section we present the application of the proposed integral operator to the Cauchy-type of fractional ordinary differential and partial differential equations. We will start with the fractional ordinary differential equation. Here we consider the Cauchytype equation of the following form:

$$
D_{r r}^{\alpha} \Phi(r)+\frac{1}{r^{n}} \Phi(r)=0, \quad l-1<\alpha \leq l .
$$

To solve the above equation, we apply on both sides the new integral transform of order $n$ to obtain the following:

$$
(-1)^{n} \frac{d^{n}}{d s^{n}} D_{r r}^{\alpha} \Phi(s)+\Phi(s)=0
$$

The new integral transform has gotten rid of the singularity; the new equation is just an ordinary fractional differential equation, which can be solved with, for instance, the homotopy decomposition method. Let us find the exact solution of the above equation for $n=1$ given below as

$$
D_{r r}^{\alpha} \Phi(r)+\frac{1}{r} \Phi(r)=0, \quad l-1<\alpha \leq l
$$

We will make use of the new integral transform to derive analytical solution of (34). Applying the new transform of order 1 on both sides of the above equation, we obtain the following expression:

$$
\begin{gathered}
\frac{d[L(\Phi)(s)]}{d s}+\left(\frac{\alpha}{s}+\frac{1}{s^{\alpha}}\right)(L(\Phi)(s)) \\
=\sum_{m=2}^{l} d_{m}(m-1) s^{m-2-\alpha},
\end{gathered}
$$

where $d_{m}=D_{0^{+}}^{\alpha-m} \Phi\left(0^{+}\right)(m=2, \ldots, l)$. Now, one can derive the solution of the ordinary order differential equation with respect to the Laplace transform of $\Theta(s)=L(\Phi(r))$ :

$$
\begin{aligned}
\Theta(s)= & s^{-\alpha} \exp \left[-\frac{s^{1-\alpha}}{1-\alpha}\right] \\
& \times\left[a_{1}+\sum_{m=2}^{l} d_{m}(m-1) \int s^{m-2} \exp \left[-\frac{s^{1-\alpha}}{1-\alpha}\right] d s\right],
\end{aligned}
$$

with $a_{1}$ an arbitrary real constant that will be obtained via the initial condition. We next expand the exponential function in the integrand in a series, and using term-by-term integration, we arrive at the following expression:

$$
\Theta(s)=c \Theta_{1}(s)+\sum_{m=2}^{l} d_{m}(m-1) \Theta_{m}^{*}(s)
$$

with of course

$$
\begin{gathered}
\Theta_{1}(s)=s^{-\alpha} \exp \left[-\frac{s^{1-\alpha}}{1-\alpha}\right], \\
\Theta_{m}^{*}(s)=s^{-\alpha} \exp \left[\frac{s^{1-\alpha}}{\alpha-1}\right] \\
\\
\times \sum_{j=0}^{\infty}\left(\frac{1}{1-\alpha}\right)^{j} \frac{s^{(1-\alpha) j+m-1}}{[(1-\alpha) j+m-1] j !} .
\end{gathered}
$$

Now, applying the inverse Laplace transform on $\Theta_{1}(s)$ and using the fact that

$$
s^{-[\alpha+(\alpha-1) j]}=L\left[\frac{r^{\alpha+(\alpha-1) j-1}}{\Gamma(\alpha+(\alpha-1) j)}\right]
$$

we obtain

$$
\Phi_{1}(r)=r^{\alpha-1} \mathrm{o} \Psi_{1}\left[(\alpha, \alpha-1) \mid \frac{x^{\alpha-1}}{\alpha-1}\right]
$$

with $o \Psi_{1}[]$ the generalized Wright function for $p=1$ and $q=2$ [34-37]. We next expand the exponential function $\exp \left[-s^{1-\alpha} /(1-\alpha)\right]$ in power series, multiplying the resulting two series; in addition to this if we consider the number $b_{k}(\alpha, m)$ defined for $\alpha>0, m=2, \ldots l(\alpha \neq(p+m-1) / p, p \notin$ $\mathbb{N})$, and $k \in \mathbb{N}_{0}$,

$$
b_{k}(\alpha, m)=\sum_{p, j=0, \ldots, k, p+j=k}^{l} \frac{(-1)^{q}}{p ! j !(1-\alpha) q+m-1} .
$$

The above family of number possesses satisfies the following recursive formula:

$$
\frac{b_{k}(\alpha, m)}{b_{k+1}(\alpha, m)}=\frac{\alpha-m}{\alpha-1}+k
$$

which produces the explicit expression for $b_{k}(\alpha, m)$ in the form of

$$
b_{k}(\alpha, m)=\frac{\Gamma[(\alpha-m) /(\alpha-1)]}{(m-1) \Gamma[((\alpha-m) /(\alpha-1))+k]}, \quad k \in \mathbb{N}_{0}
$$

Now having the above expression on hand, we can derive that

$$
\begin{aligned}
\Theta_{m}^{*}(s)= & s^{m-\alpha-1}\left(\sum_{j=0}^{\infty}\left(\frac{1}{1-\alpha}\right)^{j} \frac{s^{(1-\alpha) p}}{p !}\right) \\
& \times\left(\sum_{p=0}^{\infty}\left(\frac{1}{1-\alpha}\right)^{p} \frac{(-1)^{p}}{[(1-\alpha) p+m-1]} \frac{s^{(1-\alpha) j}}{p !}\right) \\
= & \sum_{k=0}^{\infty} b_{k}(\alpha, m)\left(\frac{1}{1-\alpha}\right)^{k} \\
& \times s^{(1-\alpha) k+m-\alpha-1} \quad(m=2, \ldots, l) .
\end{aligned}
$$


However, remembering (40) with $\beta=(\alpha-1) k+\alpha+1-m$, we can further derive the following expression for $\Phi_{m}^{*}(r)$ as

$$
\begin{aligned}
\Phi_{m}^{*}(r)=\sum_{k=0}^{\infty} b_{k}(\alpha, m)\left(\frac{1}{1-\alpha}\right)^{k} \\
\quad \times \frac{\Gamma(k+1)}{\Gamma[\alpha+1-m+(\alpha-1) k]} \frac{x^{(\alpha-1) k+\alpha-m}}{k !}
\end{aligned}
$$

or in the simplified version we have

$$
\Phi_{m}^{*}(r)=\frac{\Gamma[(\alpha-m) /(\alpha-1)]}{(m-1)} \Phi_{m}(r),
$$

where

$$
\begin{aligned}
& \Phi_{m}(r) \\
& \quad=r^{\alpha-m}{ }_{1} \Psi_{2} \\
& \quad \times\left[\begin{array}{r}
(1,1) \\
\left.(\alpha+1-m, \alpha-1),\left(\frac{\alpha-m}{\alpha-1}, 1\right) \mid \frac{r^{\alpha-1}}{\alpha-1}\right] .
\end{array}\right.
\end{aligned}
$$

It follows that the solution of the Cauchy-type equation is in the form of

$$
\begin{aligned}
& \Phi(r) \\
& =a_{1} r^{\alpha-1} \circ \Psi_{1}\left[(\alpha, \alpha-1) \mid \frac{x^{\alpha-1}}{\alpha-1}\right] \\
& \quad+a_{2} \sum_{m=2}^{l} b_{m}(m-1) \frac{\Gamma[(\alpha-m) /(\alpha-1)]}{(m-1)} r^{\alpha-m} \Psi_{2} \\
& \quad \times\left[(\alpha+1-m, \alpha-1),\left(\frac{\alpha-m}{\alpha-1}, 1\right) \mid \frac{r^{\alpha-1}}{\alpha-1}\right] .
\end{aligned}
$$

We will examine the solution of the following fractional partial differential equation of the following form:

$$
{ }_{0}^{C} D_{t}^{\alpha} u(x, t)=\frac{1}{x} \frac{\partial^{2} u(x, t)}{\partial x^{2}}, \quad 0<\alpha \leq 1,
$$

with initial and boundary conditions of the form

$$
\begin{gathered}
u(x, 0)=0, \quad u\left(x_{0}, t\right)=h(t), \\
\partial_{x} u(0, t)=u(0, t)=0(t \geq 0) .
\end{gathered}
$$

To solve the above problem, the first step consists of applying the new integral transform on both sides of (49) to obtain

$$
\partial_{s}{ }_{0}^{C} D_{t}^{\alpha} U(s, t)=-s^{2} U(s, t),
$$

where $s$ is the Laplace variable. The next step in this derivation is to apply the Fourier transform in time to obtain

$$
(i p)^{\alpha} \partial_{s} U_{1}(s, p)=-s^{2} U_{1}(s, p),
$$

where $p$ is the Fourier variable. It follows that the solution of the above equation is simply given as

$$
U_{1}(s, p)=c(p) \exp \left[-\frac{s^{3}}{3}(i p)^{-\alpha}\right] .
$$

The next step is to put exponential function in series form as follows

$$
\begin{aligned}
\exp \left[-\frac{s^{3}}{3}(i p)^{-\alpha}\right] \\
=\sum_{k=0}^{\infty} \frac{\left(\left(-s^{3} / 3\right)(i p)^{-\alpha}\right)^{k}}{k !}=\sum_{k=0}^{\infty} \frac{\left(-s^{3} / 3\right)^{k}(i p)^{-k \alpha}}{k !} .
\end{aligned}
$$

Then, we first apply the inverse Laplace in both sides of the above equation to obtain

$$
U_{1}(x, p)=L^{-1}\left(c(p) \sum_{k=0}^{\infty} \frac{\left(-s^{3} / 3\right)^{k}(i p)^{-k \alpha}}{k !}\right) .
$$

Making use of the linearity to the inverse Laplace transform, we obtain

$$
U_{1}(x, p)=\sum_{k=0}^{\infty} \frac{L^{-1}\left[\left(-s^{3} / 3\right)^{k}\right] c(p)(i p)^{-k \alpha}}{k !} .
$$

And finally making use of the inverse Fourier transform and its linearity, we obtain

$$
u(x, t)=\sum_{k=0}^{\infty} \frac{L^{-1}\left[\left(-s^{3} / 3\right)^{k}\right] F^{-1}\left[c(p)(i p)^{-k \alpha}\right]}{k !} .
$$

This, produces the solution of (49).

\section{Conclusion}

We introduced a new integral operator transform. We presented its existence and uniqueness. We presented some properties and its application for solving some kind of ordinary and partial fractional differential equations that arise in many fields of sciences.

\section{Conflict of Interests}

The authors declare that they have no conflict of interests.

\section{Authors' Contribution}

A. Atangana wrote the first draft and A. Kilicman corrected the final version. All authors read and approved the final draft.

\section{Acknowledgments}

The authors would like to thank the referee for some valuable comments and helpful suggestions. Special thanks go to the editor for his valuable time spent to evaluate this paper. 


\section{References}

[1] A. D. Polyanin and A. V. Manzhirov, Handbook of Integral Equations, CRC Press, Boca Raton, Fla, USA, 1998.

[2] R. K. M. Thambynayagam, The Diffusion Handbook: Applied Solutions for Engineers, McGraw-Hill, New York, NY, USA, 2011.

[3] M. Hazewinkel, "Integral transform," in Encyclopedia of Mathematics, Springer, 2001.

[4] B. Boashash, Time-Frequency Signal Analysis and Processing: A Comprehensive Reference, Elsevier Science, Oxford, UK, 2003.

[5] S. Bochner and K. Chandrasekharan, Fourier Transforms, Princeton University Press, Princeton, NJ, USA, 1949.

[6] R. N. Bracewell, the Fourier Transform and Its Applications, McGraw-Hill, Boston, Mass, USA, 3rd edition, 2000.

[7] G. A. Campbell and R. M. Foster, Fourier Integrals for Practical Applications, D. Van Nostrand Company, New York, NY, USA, 1948.

[8] E. U. Condon, "Immersion of the Fourier transform in a continuous group of functional transformations," Proceedings of the National Academy of Sciences of the USA, vol. 23, pp. 158-164, 1937.

[9] J. Duoandikoetxea, Fourier Analysis, vol. 29, The American Mathematical Society, Providence, RI, USA, 2001.

[10] L. Grafakos, Classical and Modern Fourier Analysis, PrenticeHall, 2004.

[11] E. Hewitt and K. A. Ross, Abstract Harmonic Analysis. Vol. II: Structure and Analysis for Compact Groups. Analysis on Locally Compact Abelian Groups, Springer, New York, NY, USA, 1970.

[12] L. Schwartz, "Transformation de Laplace des distributions," Seminaire Mathématique de l'Université de Lund, vol. 1952, pp. 196-206, 1952 (French).

[13] A. Atangana and A. Kilicman, "Analytical solutions of the spacetime fractional derivative of advection dispersion equation," Mathematical Problems in Engineering, vol. 2013, Article ID 8531279, 2013.

[14] W. M. Siebert, Circuits, Signals, and Systems, MIT Press, Cambridge, Mass, USA, 1986.

[15] A. Atangana, "A note on the triple laplace transform and its applications to some kind of third-order differential equation," Abstract and Applied Analysis, vol. 2013, Article ID 769102, 10 pages, 2013.

[16] D. V. Widder, "What is the Laplace transform?" The American Mathematical Monthly, vol. 52, pp. 419-425, 1945.

[17] J. Williams, Laplace Transforms (Problem Solvers), vol. 10, George Allen and Unwin, 1973.

[18] P. Flajolet, X. Gourdon, and P. Dumas, "Mellin transforms and asymptotics: harmonic sums," Theoretical Computer Science, vol. 144, no. 1-2, pp. 3-58, 1995.

[19] J. Galambos and I. Simonelli, Products of Random Variables: Applications to Problems of Physics and to Arithmetical Functions, vol. 4, Marcel Dekker, New York, NY, USA, 2004.

[20] G. K. Watugala, "Sumudu transform: a new integral transform to solve differential equations and control engineering problems," International Journal of Mathematical Education in Science and Technology, vol. 24, no. 1, pp. 35-43, 1993.

[21] S. Weerakoon, "Application of Sumudu transform to partial differential equations," International Journal of Mathematical Education in Science and Technology, vol. 25, no. 2, pp. 277-283, 1994.
[22] M. G. M. Hussain and F. B. M. Belgacem, "Transient solutions of Maxwell's equations based on sumudu transform," Progress in Electromagnetics Research, vol. 74, pp. 273-289, 2007.

[23] F. Oberhettinger and L. Badii, Tables of Laplace Transforms, Springer, Berlin, Germany, 1973.

[24] V. A. Ditkin and A. P. Prudnikov, Integral Transforms and Operational Calculus, Pergamon Press, Oxford, UK, 1965.

[25] W. Balser, From Divergent Power Series to Analytic Functions, vol. 1582, Springer, Berlin, Germany, 1994.

[26] A. Atangana and A. Kilicma, "The use of sumudu transform for solving certain nonlinear fractional heat-like equations," Abstract and Applied Analysis, vol. 2013, Article ID 737481, p. $12,2013$.

[27] S. Weerakoon, "The "Sumudu transform" and the Laplace transform: reply," International Journal of Mathematical Education in Science and Technology, vol. 28, no. 1, p. 160, 1997.

[28] M. Y. Ongun, "The Laplace Adomian Decomposition Method for solving a model for HIV infection of $\mathrm{CD}^{+} \mathrm{T}$ cells," Mathematical and Computer Modelling, vol. 53, no. 5-6, pp. 597-603, 2011.

[29] A. Atangana, "New class of boundary value problems," Information Sciences Letters, vol. 1, no. 2, pp. 67-76, 2012.

[30] A. Atangana and J. F. Botha, "Analytical solution of groundwater flow equation via homotopy decomposition method," Journal of Earth Science and Climatic Change, vol. 3, article 115, 2012.

[31] A. Atangana and A. Secer, "The time-fractional coupledKorteweg-de-Vries equations," Abstract and Applied Analysis, vol. 2013, Article ID 947986, 8 pages, 2013.

[32] A. Atangana and E. Alabaraoye, "Solving a system of fractional partial differential equations arising in the model of HIV infection of $\mathrm{CD}^{+}$cells and attractor one-dimensional KellerSegel equations," in Advances in Difference Equations, vol. 2013, article 94, 2013.

[33] A. Atangana, A. Ahmed, and N. Bilick, "A generalized version of a low velocity impact between a rigid sphere and a transversely isotropic strain-hardening plate supported by a rigid substrate using the concept of non-integer derivatives," Abstract and Applied Analysis, vol. 2013, Article ID 671321, 9 pages, 2013.

[34] K. B. Oldham and J. Spanier, The Fractional Calculus, Academic Press, New York, NY, USA, 1974.

[35] I. Podlubny, Fractional Differential Equations, vol. 198, Academic Press, New York, NY, USA, 1999.

[36] K. S. Miller and B. Ross, An Introduction to the Fractional Calculus and Fractional Differential Equations, John Wiley \& Sons, New York, NY, USA, 1993.

[37] A. A. Kilbas, H. M. Srivastava, and J. J. Trujillo, Theory and Applications of Fractional Differential Equations, Elsevier, Amsterdam, The Netherlands, 2006. 


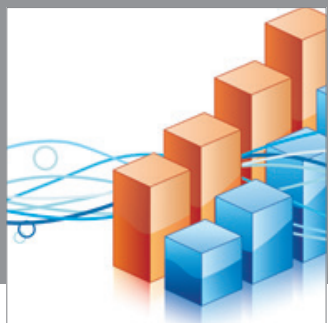

Advances in

Operations Research

mansans

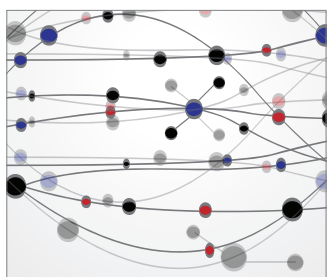

The Scientific World Journal
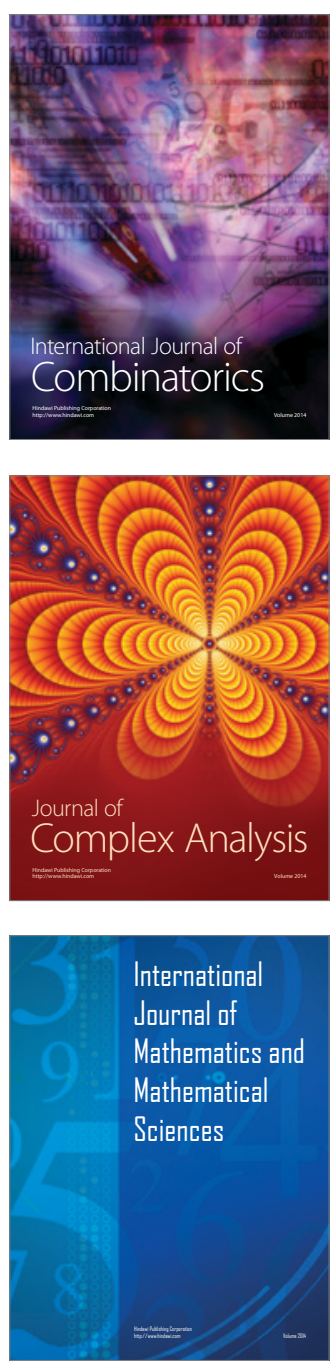
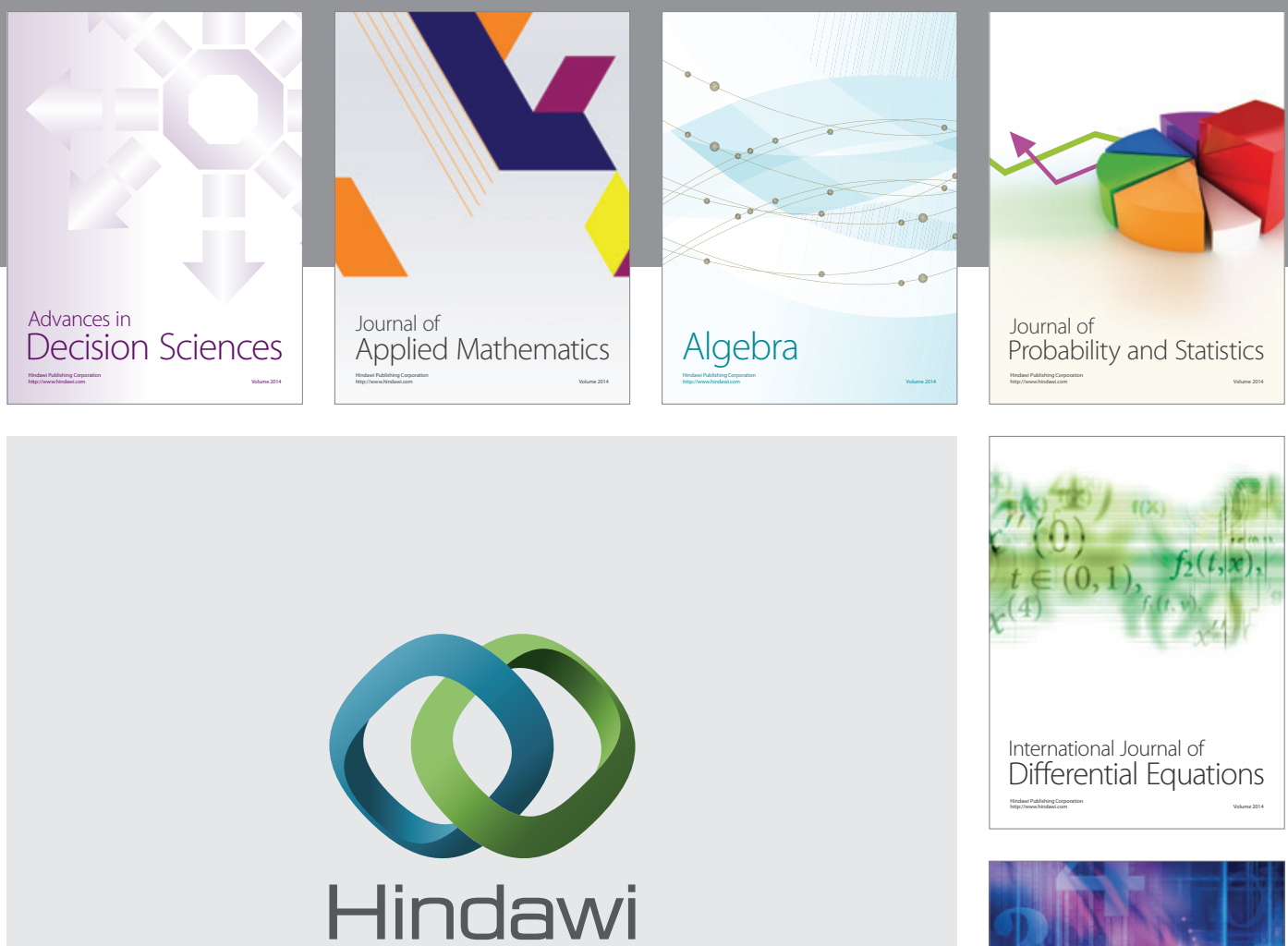

Submit your manuscripts at http://www.hindawi.com
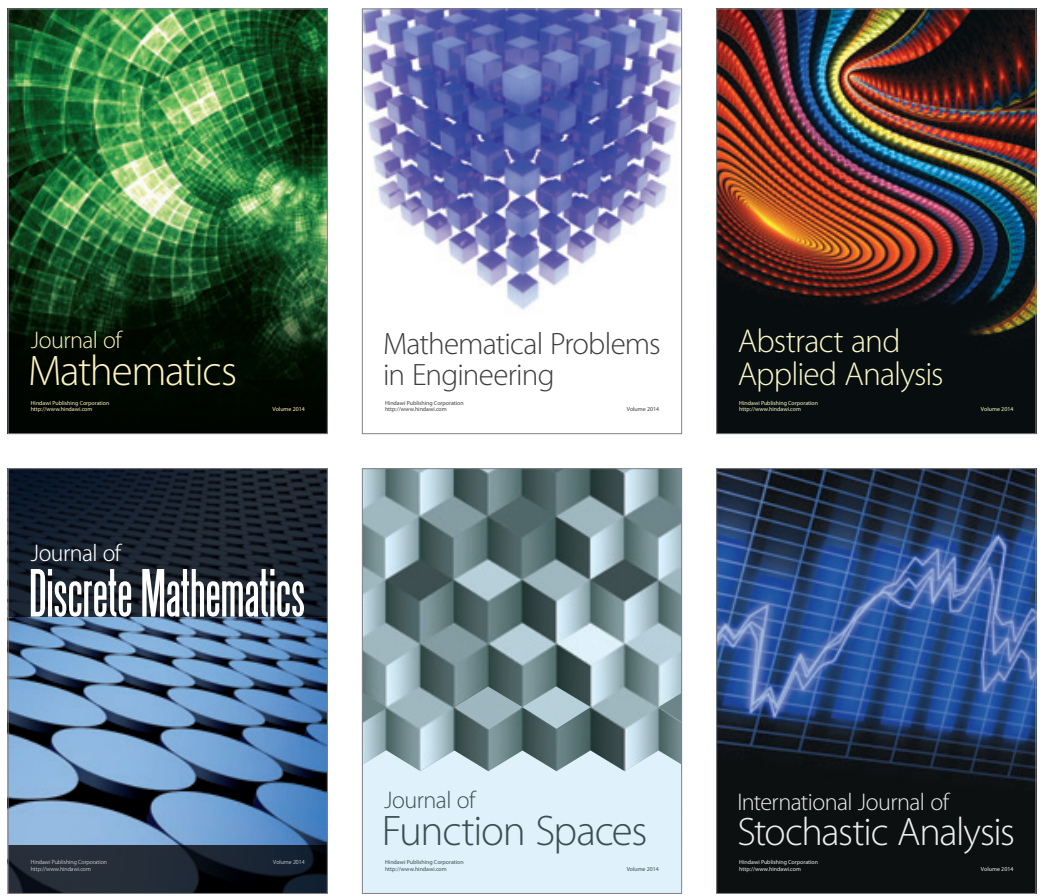

Journal of

Function Spaces

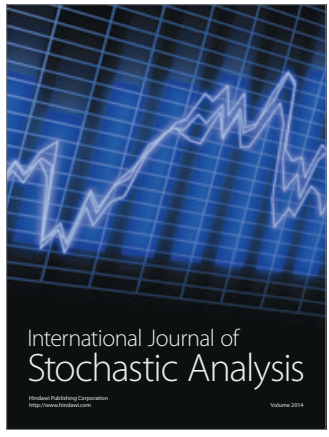

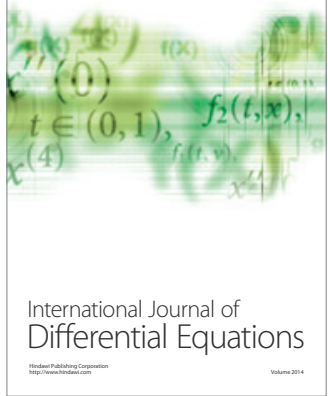
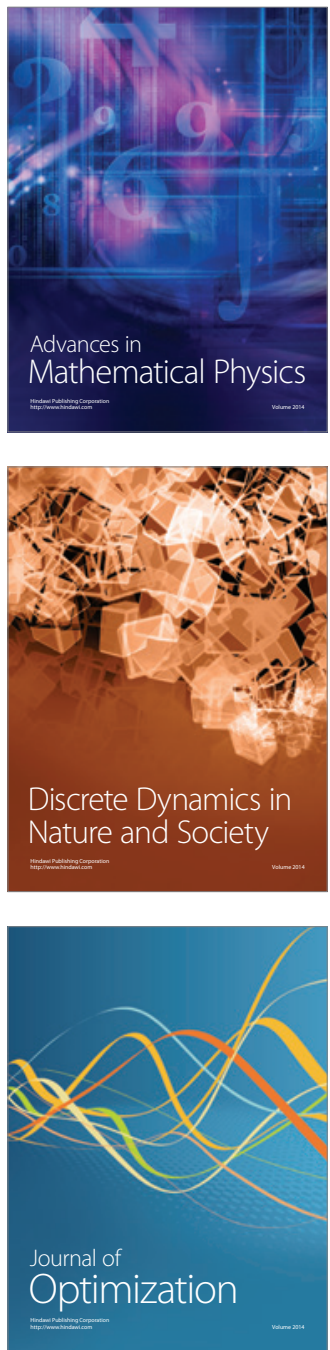